\title{
Designing Versatile Nanocomposites Based on Chitosan/Nanomaterials for Ecological Applications
}

\author{
Saira Bibi ${ }^{1}$, Shaista Taimur ${ }^{2}$, Tariq Yasin $^{2}$, Mohsan Nawaz ${ }^{1}$, Gareth J.Price ${ }^{3}$ \\ ${ }^{1}$ Department of Chemistry, Hazara University Mansehra \\ Dhodial, Pakistan \\ sairabushi@hu.edu.pk; mohsannawaz@hotmail.com \\ ${ }^{2}$ Pakistan Institute of Engineering and Applied Sciences \\ Islamabad, Pakistan \\ shaistaaimur@yahoo.co.uk; yasintariq@yahoo.com \\ ${ }^{3}$ Department of Chemistry, University of Bath \\ Bath, UK \\ g.j.price@bath.ac.uk
}

\section{Extended Abstract}

The objective of this research was to fabricate environmentally friendly chitosan based systems with tunable morphological, electrical, thermal, and mechanical characteristics. To develop such systems based on chitosan/PVA and nanomaterials a new approach was utilized. Various kinds of polymer nanocomposites were synthesized. Polymer fillers used in this work include metallic nanoparticles (silver-nanoparticles (Ag-NPs), gold-nanoparticles (Au-NPs), iron oxide $\left(\mathrm{Fe}_{3} \mathrm{O}_{4}\right)$ nanoparticles) and carbon nanotubes (CNTs). CNTs were pre-treated with ultrasonic irradiations $\left(\mathrm{HNO}_{3} / \mathrm{H}_{2} \mathrm{SO}_{4}\right.$, dilute acids) as well as by gamma irradiation. Through transmission electron microscopy (TEM) the CNTs microstructure has been investigated that explained that the two methods excellently overcame aggregation of the CNTs. The ultrasonically assisted acid treatment demonstrated the highest degree of degradation. That includes additional defect generation in the graphitic network and nanotubes shortening (at longer reaction duration or high ultrasonic intensities). Raman and FTIR spectroscopic techniques were used to investigate the nature of the surface modifications and the introduction of oxygenated species at the surface was explored. If optimum results are to be obtained, our findings explain the importance of careful control over these parameters. Gamma irradiation method offers the opportunity for a greener chemical functionalization of nanotubes.

Gamma-treated carbon nanotubes were combined along with chitosan/PVA blend. After blending polymers were crosslinked using crosslinker (silane, TEOS). Chitosan-CNTs films showed the equal distribution of $\gamma$-treated nanotubes in the blend confirmed by scanning electron micrograph (SEM). Removal of Polyaromatic hydrocarbons (PAHs) from waste water were tested by using these prepared nanocomposites. The fast removal of aforementioned pollutants and the green nature of these materials made them acceptable candidates for PAHs clean-up applications[1].

For controlled release of pesticide (Azinophos methyl) chitosan based nanocomposites having functionalized CNTs were also fabricated. Controlled release behaviour of such newly develop materials disclosed an advanced pathway for pesticide/agricultural operations. The aforementioned also needs smaller amount of drugs as an effect their dangerous impacts in surroundings can be reduced.

Chitosan nanocomposite membranes (innovative and green) having gold-NPs and carbon nanotubes were fabricated. The catalytic activity of the nanocomposites was observed for 4-nitrophenol to 4-aminophenol conversion. The existence in regard to functionalized nanotubes enhanced the speed of the system, primarily by marking down the induction period (proved by kinetic studies). Catalytically active films fabricated from the nanocomposites can be recycled by a magnitude of several cycles without any loss of catalytic effect or mechanical integrity, opening up the feasibility of utilizing such eco-friendly, novel catalyst bed in continued flow systems[2].

Using sonochemical technique Ag-NPs were successfully prepared. Fabrication of chitosan/Ag-NPs membranes using ultrasound irradiations was performed by in situ polymerization. Our findings revealed that fillers addition enhanced the mechanical strength of chitosan. These enhanced results indirectly proves better dispersion of both fillers under ultrasonic 
treatment in polymer. The improved efficiency of these nanocomposites also used by adding iron oxide nanoparticles within the polymer to design multi-use, conveniently recyclable catalysts for methylene blue removal.

The developed materials showed quite promising properties and applications. In addition, these newly synthesized nanocomposites provide new addition in chemical synthesis, water engineering, and agricultural field and membrane separation technologies.

\section{References}

[1] S. Bibi, T. Yasin, S. Hassan, M. Riaz, and M. Nawaz, "Chitosan/CNTs green nanocomposite membrane: Synthesis, swelling and polyaromatic hydrocarbons removal," Mater. Sci. Eng.C. vol. 46, pp. 359-365, 2015.

[2] S. Bibi, G. J. Price, T. Yasin, and M. Nawaz, "Eco-friendly synthesis and catalytic application of chitosan/gold/carbon nanotube nanocomposite films," RSC Adv. vol. 6, pp. 60180-60186, 2016. 